\title{
The Effectiveness of Mapping Biopsy in Patients with Extramammary Paget's Disease
}

\author{
Byung Jun Kim, Shin Ki Park, Hak Chang \\ Department of Plastic and Reconstructive Surgery, Seoul National University Hospital, Seoul National University College of Medicine, Seoul, \\ Korea
}

Background Extramammary Paget's disease (EMPD) is an intraepithelial carcinoma usually occurring on the skin or mucosa of the perineum. Clinically, it resembles eczema or dermatitis, and misdiagnosis and treatment delays are common. The treatment of choice for EMPD is a wide excision with adequate margins. Wide excision with intraoperative frozen biopsy and Mohs micrographic surgery are common methods; however, these are associated with a high recurrence rate and long operation time, respectively.

Methods Between January 2010 and June 2013, 21 patients diagnosed with EMPD underwent mapping biopsy. Biopsy specimens were collected from at least 10 areas, $2 \mathrm{~cm}$ from the tumor margin. When the specimens were positive for malignancy, additional mapping biopsy was performed around the biopsy site of the positive result, and continued until no cancer cells were found. Based on the results, excision margins and reconstruction plans were established preoperatively.

Results The patients (18 male, 3 female) had a mean age of 66.5 years (range, $50-82$ years). Almost all cases involved in the perineal area, except one case of axillary involvement. Permanent biopsy revealed one case (4.8\%) of positive cancer cells on the resection margin, in which additional mapping biopsy and re-operation was performed. At the latest follow-up (mean, 27.4 months; range, 12-53 months), recurrence had not occurred.

Conclusions Preoperative mapping biopsy enables accurate resection margins and a preoperative reconstructing plan. Additionally, it reduces the operation time and risk of recurrence. Accordingly, it represents an effective alternative to Mohs micrographic surgery and wide excision with intraoperative frozen biopsy.

Keywords Paget disease, extramammary / Mohs surgery / Frozen sections
Correspondence: Hak Chang Department of Plastic and Reconstructive Surgery, Seoul National University Hospital, Seoul National University College of Medicine, 103 Daehak-ro, Jongno-gu, Seoul 110-744, Korea

Tel: +82-2-2072-3086

Fax: +82-2-747-5130

E-mail: hchang@snu.ac.kr

This research was supported by the Basic Science Research Program through the National Research Foundation of Korea (NRF) funded by the Ministry of Education, Science and Technology (R11-2005-065).

No potential conflict of interest relevant to this article was reported.

Received: 10 Jun 2014 • Revised: 3 Jul 2014 • Accepted: 14 Jul 2014

pISSN: 2234-6163 • elSSN: 2234-6171 • http://dx.doi.org/10.5999/aps.2014.41.6.753 • Arch Plast Surg 2014;41:753-758

\section{INTRODUCTION}

Extramammary Paget's disease (EMPD) is a rare intraepithelial slow-growing malignancy that is difficult to distinguish from other skin lesions such as eczema, contact dermatitis, or Bowen's disease, clinically. Accordingly, misdiagnosis often occurs, re- sulting in proper treatment being delayed. Although EMPD is slow growing, it frequently undergoes regional or distant metastasis through direct dermal invasion and lymphatic spread [1], and such delays in treatment may hence be critically harmful to the patients $[2,3]$.

Therapeutic modalities suggested for EMPD include chemo- 
therapy, radiotherapy, local laser treatment, and topical cytotoxic agent application. However, wide excision with an adequate margin $(1.5-3 \mathrm{~cm})$ is generally the recommended method. The optimal surgical resection margin is often difficult to establish, owing to the irregular and geographically shaped margins of EMPD, and the fact that tumor invasion may precede the visible changes of the skin lesion [4]. To solve this problem, Mohs micrographic surgery (MMS) or intraoperative frozen biopsy is usually performed to establish the surgical excision margin. During MMS, since the operator can directly observe the margin of the lesion using a microscope, normal tissue can be preserved as much as possible, while the risk of local recurrence is reduced [5]. However, due to the thorough observation and highly specialized technique, MMS is associated with a long operation time, which is a major drawback of the procedure. On the other hand, intraoperative frozen biopsy is a relatively easy technique; however, this method is also associated with an elongated operation time for intraoperative biopsy reading, as well as with a high false negative rate. A prolonged operation time might have significant effects on patient morbidity, and increase the overall medical costs, whereas false negative results may lead to imperfect resections. According to previous studies, the false negative rate of frozen biopsy ranges $10.4 \%$ to $13.2 \%$, making the local recurrence rate higher than $16 \%[1,6,7]$. In the present study, we performed preoperative mapping biopsy in EMPD patients in order to overcome these problems, and to obtain accurate resection margins and a preoperative reconstructing plan.

\section{Fig. 1. Preoperative mapping biopsy at OPD}

For 57-year-old male patient, punch biopsy was performed at the margin of $2 \mathrm{~cm}$ away from the lesion. OPD, outpatient department.

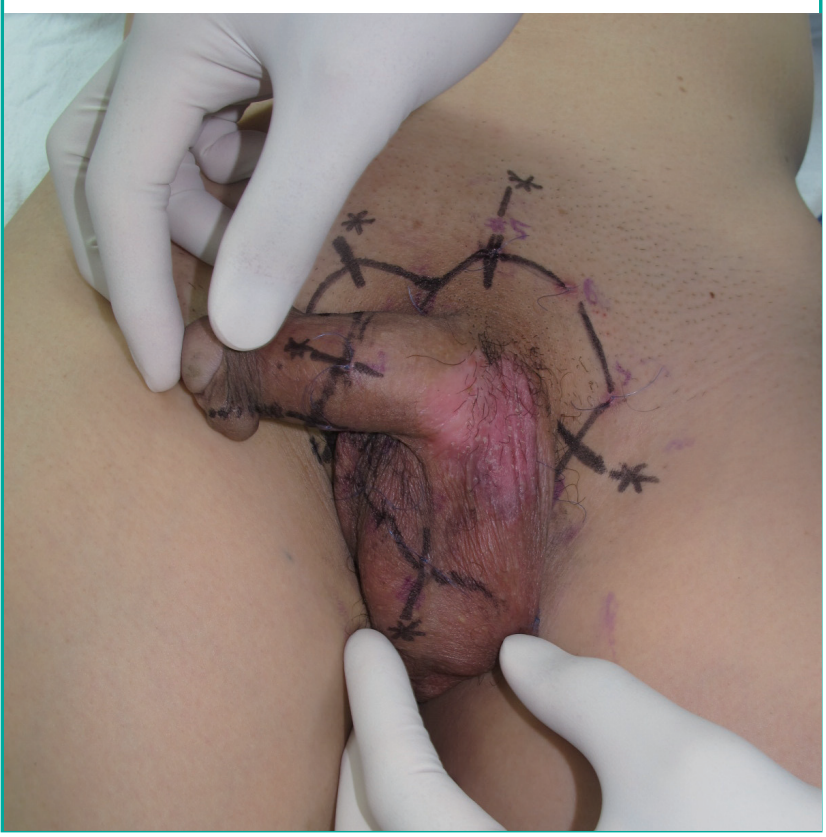

\section{METHODS}

Between January 2010 and June 2013, 21 EMPD patients, those histologically confirmed by dermatologist, were referred to our department. All patients underwent preoperative mapping biopsy at our outpatient clinic. The outline of the grossly visible EMPD lesion was marked, and punch biopsies were conducted on at least 10 sites, $2 \mathrm{~cm}$ from the outline (Fig. 1). The number of mapping biopsy sites were adjusted depending on the size and shape of the skin lesion. Numbers were given to each biopsy site in a clockwise fashion to facilitate the communication with the pathologist. If the skin laxity was large, such as for the scrotum, mapping biopsy was performed while the skin was unfolded by gentle traction. The biopsy wound sites were repaired with 4-0 nylon sutures, not only for wound healing, but also for helping to identify the biopsy sites later. On the next visit to confirm the biopsy result, the resection margin was determined if the results revealed no tumor cells in the entire specimen. If the punch biopsy results were positive for EMPD, additional biopsies around the previous biopsy site with an additional $2 \mathrm{~cm}$ safety margin were performed. The additional biopsy was usually performed at 4-6 points around the site of positive result, and we repeated this procedure until the entire specimen showed negative tumor cells (Fig. 2).

After the end of the mapping biopsy, we made a preoperative reconstruction plan considering the range of excision. In male patients, we decided on a scrotal flap for the scrotum and a split-

Fig. 2. Schematic flow chart of EMPD patient

Patients who diagnosed EMPD were repeated mapping biopsy until the all specimen showed negative of tumor cells. EMPD, extramammary Paget's disease; f/u, follow up.

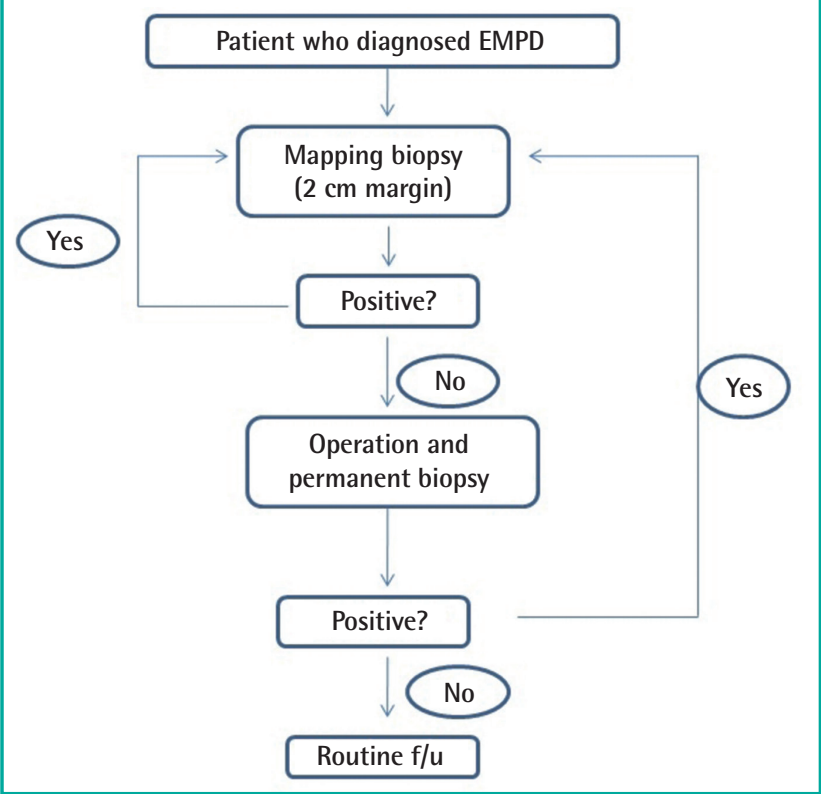


thickness skin graft (STSG) for the penile shaft. In female patients, the defect was usually covered with a local advancement flap and STSG.

\section{RESULTS}

The clinical characteristics and demographics of the patients are summarized in Table 1. Mapping biopsy was performed in 21 patients ( 18 male and 3 female patients) with a mean age at the time of operation of 66.5 years (range, 50-82 years). The scrotum along with the penile shaft was involved in 13 male patients, while exclusive scrotal involvement was shown in 4 patients and one male patient suffered EMPD of the axilla. The vulva was the predominant site of involvement in all female patients. Resection margins could be immediately obtained with mapping biopsy in fifteen patients, while 4 and 2 patients underwent 2 and 3 biopsies, because of positive results of the initial mapping biopsy. The average number of biopsies needed to achieve the final resection margin was 1.4. The patients had to visit the outpatient department (OPD) an average of 2.4 times to undergo the mapping biopsy and to confirm the results. And it took average 2.7 days to get the pathological result after the mapping biopsy. The mean number of sites biopsied was 12.7 (range, 10$16)$, and secondary and tertiary biopsies were performed at an average of 4.4 sites.
All patients underwent wide excision of the EMPD along with the previously determined resection margin, at the level of the superficial fascia. A number of deep tissues were sent to the pathologist intraoperatively to check remnant malignancy, but the results revealed negative in all cases. The specimen was sent to the pathologist for permanent biopsy (Fig. 3). The average tumor size was $48.8 \mathrm{~cm}^{2}$, and the average values of the long and short axes were $7.6 \mathrm{~cm}$ and $5.7 \mathrm{~cm}$, respectively. Scrotal flap and

\section{Fig. 3. Paget's cells at permanent biopsy}

Cells (black arrow) have pleomorphic nuclei, prominent nucleoli, and abundant cytoplasm $\left(H \& E_{1} \times 100\right)$.

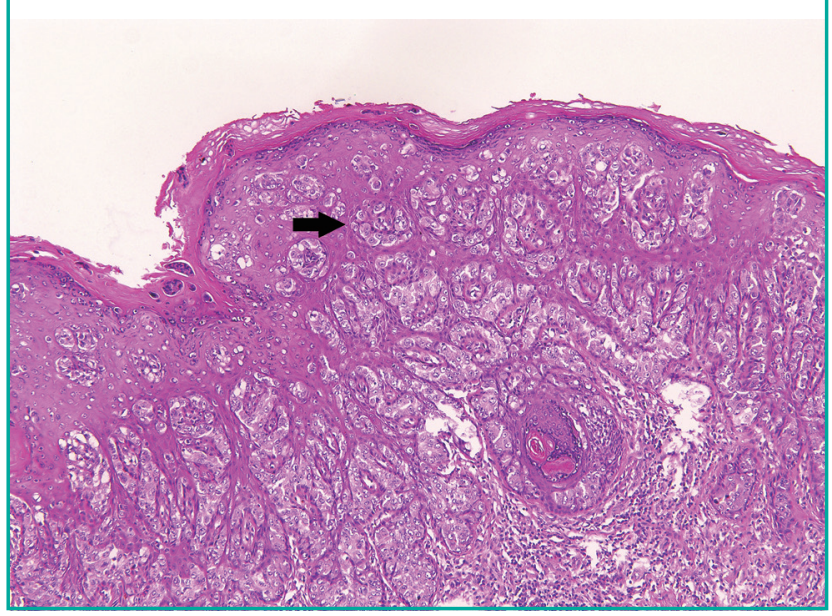

\section{Table 1. Summary of the clinical characteristics and demographics of the patients}

\begin{tabular}{|c|c|c|c|c|c|c|c|c|c|}
\hline Case & $\begin{array}{c}\text { Sex/ } \\
\text { Age (yr) }\end{array}$ & $\begin{array}{l}\text { Tumor } \\
\text { size }\left(\mathrm{cm}^{2}\right)\end{array}$ & Area & $\begin{array}{l}\text { Lymph node metastasis/ } \\
\text { Internal malignancy }\end{array}$ & $\begin{array}{l}\text { Reconstruction } \\
\text { methods }\end{array}$ & $\begin{array}{l}\text { Permanent biopsy/ } \\
\text { Depth of invasion }\end{array}$ & $\begin{array}{l}\text { Follow-up } \\
\text { period (mo) }\end{array}$ & $\begin{array}{l}\text { No. of OPD visit for } \\
\text { mapping biopsy }\end{array}$ & $\begin{array}{l}\text { No. of mapping } \\
\text { biopsy site }\end{array}$ \\
\hline 1 & $\mathrm{~F} / 70$ & $11 \times 8$ & $V$ and $P A$ & $\mathrm{~N}$ & L and STSG & N/NR & 53 & 2 & $12+5$ \\
\hline 2 & $\mathrm{~F} / 50$ & $11 \times 9$ & $V$ and $P A$ & $\mathrm{~N}$ & $\mathrm{~L}$ and STSG & $\mathrm{N} / \mathrm{DI}$ & 40 & 1 & 12 \\
\hline 3 & $\mathrm{~F} / 76$ & $12 \times 10.5$ & $V$ and $P A$ & $\mathrm{~N}$ & $\mathrm{~L}$ and STSG & $\mathrm{N} / \mathrm{DI}$ & 40 & 1 & 15 \\
\hline 4 & $M / 82$ & $13.2 \times 10$ & PS and $S$ & $\begin{array}{l}\text { P (colon cancer, inguinal } \\
\text { LN metastasis) }\end{array}$ & SF and STSG & N/ND & 37 & 3 & $10+3+4$ \\
\hline 5 & $M / 66$ & $7.5 \times 4.0$ & PS and $S$ & $\mathrm{~N}$ & SF and STSG & N/NR & 34 & 1 & 10 \\
\hline 6 & $\mathrm{M} / 70$ & $8.1 \times 5.5$ & A & $\mathrm{N}$ & STSG & $\mathrm{N} / \mathrm{DI}$ & 32 & 1 & 13 \\
\hline 7 & $\mathrm{M} / 73$ & $1.1 \times 1.0$ & S & $\mathrm{N}$ & SF & $\mathrm{N} / \mathrm{DI}$ & 30 & 1 & 10 \\
\hline 8 & $\mathrm{M} / 72$ & $6.5 \times 6.4$ & S & P (cholangiocarcinoma) & SF & N/DI & 30 & 2 & $14+8$ \\
\hline 9 & $M / 64$ & $6.5 \times 4.3$ & PS and S & $\mathrm{N}$ & SF and STSG & $\mathrm{N} / \mathrm{DI}$ & 29 & 1 & 14 \\
\hline 10 & $M / 59$ & $4.8 \times 2.9$ & S & N & SF & $\mathrm{N} / \mathrm{DI}$ & 26 & 1 & 10 \\
\hline 11 & $M / 57$ & $11.8 \times 6.3$ & PS and $S$ & $\mathrm{~N}$ & SF and STSG & $\mathrm{N} / \mathrm{DI}$ & 26 & 3 & $14+4+4$ \\
\hline 12 & $M / 63$ & $5.8 \times 3.7$ & S & $\mathrm{N}$ & SF & N/DI & 26 & 1 & 12 \\
\hline 13 & $M / 60$ & $5.2 \times 5.0$ & PS and $S$ & $\mathrm{~N}$ & SF and STSG & $\mathrm{N} / \mathrm{DI}$ & 26 & 1 & 12 \\
\hline 14 & $\mathrm{M} / 71$ & $6.7 \times 5.4$ & PS and $S$ & $\mathrm{~N}$ & SF and STSG & N/DI & 26 & 1 & 12 \\
\hline 15 & $\mathrm{M} / 74$ & $5.2 \times 3.8$ & PS and $S$ & $\mathrm{~N}$ & SF and STSG & N/NR & 25 & 1 & 15 \\
\hline 16 & $\mathrm{M} / 71$ & $14.4 \times 7.9$ & $\begin{array}{l}\text { PS and S; } \\
\text { S }\end{array}$ & $\mathrm{N}$ & $\begin{array}{l}\text { SF and STSG } \\
\text { SF }\end{array}$ & $\begin{array}{l}\text { P/DI } \\
\text { N/NR }\end{array}$ & $\begin{array}{l}23 \\
22\end{array}$ & $\begin{array}{l}2 \\
1\end{array}$ & $\begin{array}{l}12+4 \\
10\end{array}$ \\
\hline 17 & $M / 54$ & $9.0 \times 4.0$ & PS and $S$ & $\mathrm{~N}$ & SF and STSG & N/ND & 17 & 1 & 12 \\
\hline 18 & $M / 69$ & $7.0 \times 4.2$ & PS and $S$ & $\mathrm{P}$ (inguinal LN metastasis) & SF and STSG & $\mathrm{N} / \mathrm{SI}$ & 16 & 1 & 12 \\
\hline 19 & $\mathrm{M} / 71$ & $1.3 \times 8.7$ & PS and $S$ & $\mathrm{~N}$ & SF and STSG & N/DI & 15 & 1 & 16 \\
\hline 20 & $\mathrm{M} / 67$ & $6.4 \times 6.0$ & PS and S & $\mathrm{N}$ & SF and STSG & $\mathrm{N} / \mathrm{DI}$ & 13 & 1 & 16 \\
\hline 21 & $M / 57$ & $4.4 \times 3.2$ & PS and $S$ & $\mathrm{~N}$ & SF and STSG & N/ND & 12 & 2 & $13+3$ \\
\hline
\end{tabular}

In case 16, reoperation was conducted as a result of the positive resection margin.

OPD, outpatient department; F, female; V, vulva; PA, perineal area; N, negative; L, local advancement flap; STSG, split-thickness skin graft; NR, not recoded; DI, dermal invasion; M, male; PS, penile shaft; S, scrotum; P, positive; SF, scrotal flap; ND, no dermal invasion; A, axilla; SI, subcutaneous tissue invasion. 


\section{Fig. 4. Scrotum and penile shaft reconstruction}

Split thickness skin graft and scrotal flap techniques were used to reconstruct the scrotum and penile shaft after the wide excision.

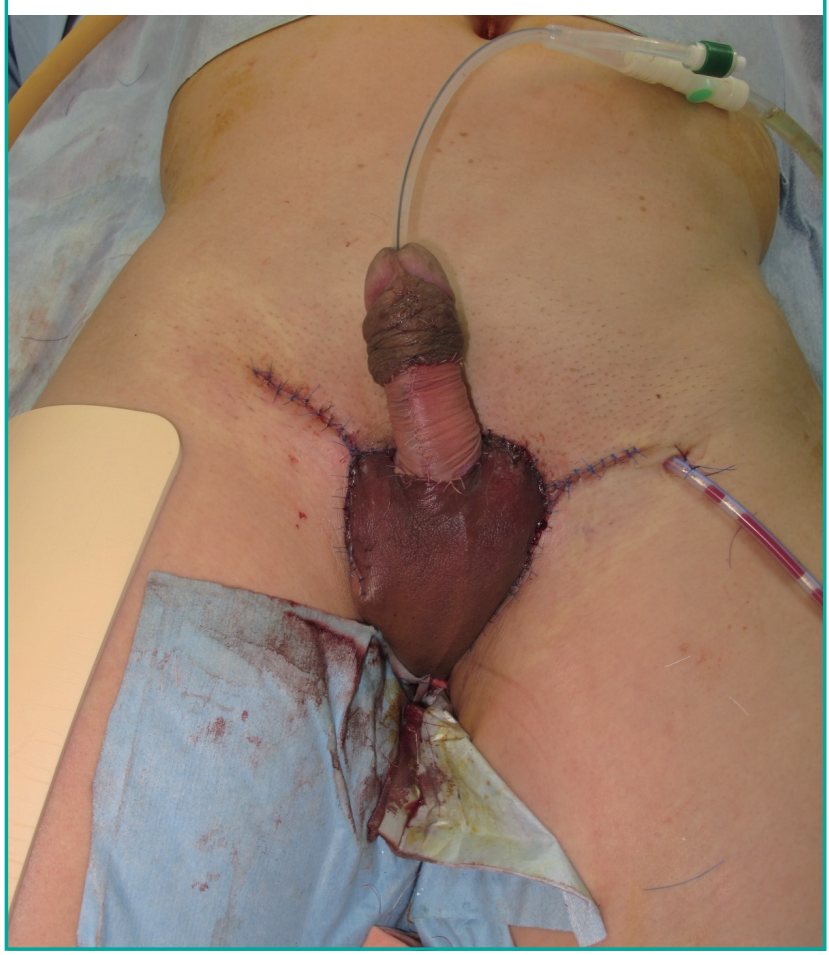

STSG (Fig. 4), scrotal flap, local advancement flap, and STSG only were performed in 13 (62\%), 4 (19\%), 3 (14\%), and 1 (5\%) patients, respectively. All female patients underwent vaginectomy or vulvectomy by gynecologist, and periurethral dissection by urologist. Groin lymph node metastasis was detected in 2 patients. These patients underwent inguinal lymph node dissection by a general surgeon, and adjuvant radiation therapy was performed in one patient. Two patients, including one who had lymph node metastasis, developed internal malignancy. Twenty patients showed free resection margins from the permanent biopsy, whereas involvement of the resection margin was found in one patient. This patient had to undergo additional mapping biopsy at the OPD, and reoperation was performed 1 month after the initial operation. The depth of invasion in permanent biopsy was found in 18 out of 21 cases. Invasion depth was confined to the epidermis in 3 cases, but 14 cases showed invasion to the dermis, and subcutaneous invasion was found in 1 case. The mean follow-up period was 27.4 months (range, 12-53 months), and none of the 21 patients, including the patient who underwent additional resection, showed any sign of recurrence.

\section{DISCUSSION}

EMPD was first described by James Paget, and usually occurs at apocrine glands-rich areas, such as the penis, scrotum, vulva, perineum, and axillae of middle-aged and older individuals $[2,3]$. It presents as reddish patches or gray-white plaques, which are eczematoid, crusty scaling, or papillomatous. It can be easily misdiagnosed as contact dermatitis, psoriasis, eczema, Bowen's disease, or tinea cruris before histological examination, owing to their similar appearance and symptoms. For this reason, proper treatment may be delayed [8]. Although EMPD is an epidermal skin cancer, dermal invasion and lymph node metastasis have been reported [9]. Furthermore, EMPD is often related with internal malignancies of the lower gastrointestinal or genitourinary tract, which requires additional examination [10]. Many conservative therapies have been introduced for EMPD, including Nd:YAG and $\mathrm{CO}_{2}$ laser treatment, 5-fluorouracil local treatment, and radiation therapy, but the results of these treatments are still controversial [11-13]. Therefore, complete surgical resection is currently recommended for definite treatment of EMPD.

EMPD usually infiltrates more than we could visually examine, making it difficult to determine the resection range, and consequently leading to a high rate of local recurrences. Zhu et al. [6] previously reported that even when a safety margin of 2 $\mathrm{cm}$ was applied, approximately $40 \%$ of permanent biopsies turned out to be margin positive. Mapping biopsy is used for skin cancers in which it is hard to determine the resection margin, such as melanoma and Bowen's disease $[14,15]$. However, to date, few studies have been conducted to evaluate the efficacy of mapping biopsy on EMPD. Kato et al. [16] reported on the clinical results of mapping biopsy in EMPD patients. They performed mapping biopsy of at least 4 points (mean, 6.3 points) per lesion, and found that $5.9 \%$ of patients experienced recurrence within 10 months. However, Paget's cells were observed in the permanent biopsies of $47 \%$ of patients, which is even higher than that of Zhu's study, and the disease is hence likely to recur at a higher rate in long-term follow up. In this study, we performed mapping biopsy at an average of 12.7 sites, $2 \mathrm{~cm}$ away from the grossly visible outline, and by repeating this procedure until all negative results were obtained, the positive rate of permanent biopsy was reduced to $4.8 \%$, and there was no tumor recurrence after an average of 27.4 months of follow up. Higher mapping resolution of our technique is thought to minimize a false negative margin. Although the safety resection margin for EMPD varies among articles, 1 to $3 \mathrm{~cm}$ is generally accepted [1]. Hatta et al. [17] reported no differences in a recurrence rate when resection margin over $2 \mathrm{~cm}$ was adopted, and we followed their opinion. It is known that the local recurrence is influenced by the primary depth of tumor [18]. Unfortunately, it was not able measure the invasion depth in the current 
method of mapping biopsy. Instead, deeper invasion beyond dermis layer in permanent biopsy was considered high risk for recurrence, and close observation was conducted in such cases.

Mapping biopsy has many advantages over conventional MMS or frozen biopsy. First, a preoperative reconstruction plan can be established; we can prepare the operation in advance, and provide the patients with more accurate information about the surgery. Second, a more accurate operation is possible. The false negative rate of punch biopsy, as determined in this study, was $4.8 \%(1 / 21)$, which is lower than that reported for frozen biopsy $(10.4 \%-13.2 \%)[1,6,7]$. Consequently, the local recurrence rate could be minimized. Third, the operation time can be shortened. According to a previous study, around 30 minutes are required for frozen biopsy in breast cancer [19], and it would take even longer to get the results for EMPD, because of the relative difficult reading of the skin cancer histology and the large specimen number needed. In cases of positive specimens, even more time is needed for the additional biopsy. The average time required for MMS has not been elucidated. For MMS in EMPD, slides are produced as an average of 2.45 times, and 20 to 30 minutes are required for each procedure. Therefore it can be assumed that it takes significantly more time for the excision, producing slides, microscopic examination, and wound repair [20,21].

However, there are also some disadvantages of mapping biopsy. The patients experience the inconvenience of visiting the OPD several times. According to our study, the average number of preoperative clinic visits was 2.4 times, and 6 patients (28.6\%) visited 3 times. However, independent of the type of treatment, EMPD patients usually require multiple clinic visits before the operation, because of work ups for other internal malignancies. Thus, we could minimize the patients' number of visits by coordinating the medical treatment schedule with other departments. Moreover, there is a concern for a surgical delay from multiple mapping biopsy, but EMPD is a slow-growing tumor, and the effect of this time interval is hence likely minimal. Multiple visits to clinician and the numbers of exam may worry patients for expensive medical cost, however, the national health insurance in Korea covers majority of the costs. Near $95 \%$ coverage allows EMPD patients pay only 11,000 KRW per each biopsy site.

In conclusion, we obtained excellent results of mapping biopsy in terms of determining the accurate preoperative resection margin and lowering the recurrence rate, and preoperative mapping biopsy can be considered an effective alternative to MMS and intraoperative frozen biopsy. Furthermore, higher preoperative mapping biopsy resolution is associated with decreased recurrence of EMPD. However, this study was limited by the relatively small number of cases $(\mathrm{n}=21)$ and short follow-up period (mean, 27.4 months). Thus, additional large-scale, long-term studies of mapping biopsy are needed in the future.

\section{REFERENCES}

1. Chan JY, Li GK, Chung JH, et al. Extramammary Paget's disease: 20 years of experience in chinese population. Int $\mathrm{J}$ Surg Oncol 2012;2012:416418.

2. Pitman GH, McCarthy JG, Perzin KH, et al. Extramammary Paget's disease. Plast Reconstr Surg 1982;69:238-44.

3. Paget J. On disease of the mammary areola preceding cancer of the mammary gland. St Bartholomew Hosp Rep 1874; 10:87-9

4. Mohs FE, Blanchard L. Microscopically controlled surgery for extramammary Paget's disease. Arch Dermatol 1979; 115:706-8.

5. O'Connor WJ, Lim KK, Zalla MJ, et al. Comparison of mohs micrographic surgery and wide excision for extramammary Paget's disease. Dermatol Surg 2003;29:723-7.

6. Zhu Y, Ye DW, Chen ZW, et al. Frozen section-guided wide local excision in the treatment of penoscrotal extramammary Paget's disease. BJU Int 2007;100:1282-7.

7. Kodama S, Kaneko T, Saito M, et al. A clinicopathologic study of 30 patients with Paget's disease of the vulva. Gynecol Oncol 1995;56:63-70.

8. Lloyd J, Flanagan AM. Mammary and extramammary Paget's disease. J Clin Pathol 2000;53:742-9.

9. Koo YT, Minn KW, Chang H. Penoscrotal extramammary Paget's disease with multiple lymph node metastasis. Arch Plast Surg 2013;40:650-2.

10. Chanda JJ. Extramammary Paget's disease: prognosis and relationship to internal malignancy. J Am Acad Dermatol 1985;13:1009-14.

11. Yoon ES, Choi JB, Han SK, et al. Treatment of extramammary paget's disease using the $\mathrm{CO} 2$ laser.J Korean Soc Plast Reconstr Surg 2000;27:169-72.

12. Bewley AP, Bracka A, Staughton RC, et al. Extramammary Paget's disease of the scrotum: treatment with topical 5-fluorouracil and plastic surgery. Br J Dermatol 1994;131:445-6.

13. Burrows NP, Jones DH, Hudson PM, et al. Treatment of extramammary Paget's disease by radiotherapy. Br J Dermatol 1995;132:970-2.

14. Dengel L, Turza K, Noland MM, et al. Skin mapping with punch biopsies for defining margins in melanoma: when you don't know how far to go. Ann Surg Oncol 2008;15: 3028-35.

15. Strauss RJ, Fazio VW. Bowen's disease of the anal and perianal area. A report and analysis of twelve cases. Am J Surg 1979;137:231-4. 
16. Kato T, Fujimoto N, Fujii N, et al. Mapping biopsy with punch biopsies to determine surgical margin in extramammary Paget's disease. J Dermatol 2013;40:968-72.

17. Hatta N, Yamada M, Hirano T, et al. Extramammary Paget's disease: treatment, prognostic factors and outcome in 76 patients. Br J Dermatol 2008;158:313-8.

18. Lai YL, Yang WG, Tsay PK, et al. Penoscrotal extramammary Paget's disease: a review of 33 cases in a 20-year experience. Plast Reconstr Surg 2003;112:1017-23.

19. Butler-Henderson K, Lee AH, Price RI, et al. Intraoperative assessment of margins in breast conserving therapy: a systematic review. Breast 2014;23:112-9.

20. Lee KY, Roh MR, Chung WG, et al. Comparison of Mohs micrographic surgery and wide excision for extramammary Paget's disease: Korean experience. Dermatol Surg 2009; 35:34-40.

21. Goldsmith LA, Katz SI, Gilchrest BA, et al. Fitzpatrick's dermatology in general medicine. 8th ed. New York: McGrawHill Medical; 2012. 